\title{
AULAS PRÁTICAS COMO ESTRATÉGIA PARA O ENSINO DE BOTÂNICA NO ENSINO FUNDAMENTAL ${ }^{1}$
}

\author{
Bruna Trindade Borges \\ Julia Dutra Vargas \\ Paula Juliane Barbosa de Oliveira \\ Silvane Vestena ${ }^{2}$
}

\begin{abstract}
RESUMO
No ensino de Ciências, a Botânica é uma das áreas com maior dificuldade de assimilação de conteúdos. Destaca-se, dentre suas causas, o desinteresse dos alunos pelo tema, a falta de aulas práticas e de materiais didáticos facilitadores. Este estudo objetivou avaliar a realização de palestras e experimentos na aprendizagem de conceitos de Botânica por discentes do Ensino Fundamental. O presente estudo foi realizado no Município de São Gabriel, Rio Grande do Sul em três Escolas Municipais. As atividades realizadas foram construção de modelos didáticos, palestras e experimentos demonstrativos sobre Botânica em laboratório e a campo, totalizando a participação de 120 alunos em cada atividade realizada. Pode-se observar, neste estudo, que as atividades realizadas foram positivas para o processo de ensinoaprendizagem, com acréscimo no conhecimento; uma vez que, o conhecimento foi significativo tanto para o discente do Ensino Fundamental como para os acadêmicos da Universidade Federal do Pampa, especialmente, para sua formação profissional, especialmente os do Curso de Licenciatura em Ciências Biológicas.
\end{abstract}

Palavras-chave: Recursos didáticos. Educação ambiental. Ensino-aprendizagem.

\section{INTRODUÇÃO}

A Botânica, enquanto estudo das plantas e área da Biologia, ao longo de sua história, concebeu teorias, gerou pensadores e sustentou formas de pensamento. Através de seu percurso histórico, fez opções, tomou caminhos e constituiu uma trajetória que influenciou e influencia a pesquisa-ciência e o ensino. $\mathrm{O}$ pensamento botânico perpassou os diferentes momentos diretamente da história, traçando um perfil específico a cada período (SILVA et al., 2015).

\footnotetext{
${ }^{1}$ Como citar este artigo: BORGES, Bruna Trindade; VARGAS, Julia Dutra; OLIVEIRA, Paula Juliane Barbosa de; VESTENA, Silvane. Aulas práticas como estratégia para o ensino de botânica no ensino fundamental. ForScience: revista científica do IFMG, Formiga, v. 7, n. 2, e00687, jul./dez. 2019. DOI: 10.29069/forscience.2019v7n2.e687.
}

2 Autor para correspondência: Silvane Vestena, e-mail: silvane.vestena@ifmg.edu.br 
A Botânica está intimamente relacionada com o cotidiano e precisa ser trabalhada de forma com que o aluno se motive e tenha vontade de participar das aulas, para isso não é preciso muito, aulas simples ou diversificadas podem tornar-se interessantes e auxiliar a compreensão do aluno em relação ao conteúdo (SILVA; TERÁN, 2018). Uma alternativa são as aulas práticas que não precisam necessariamente contemplar experimentos no laboratório e despertam em geral um grande interesse nos alunos, além de propiciar uma situação de investigação (SOUZA; KINDEL, 2014) e a utilização de modelos didáticos, que são facilitadores para uma melhor compreensão de alguns conteúdos da Botânica. Durante uma atividade prática, o docente consegue aproximar o aluno da sua realidade, trabalhando a importância que as plantas adquirem em diversas vertentes, principalmente para o planeta e para os seres humanos (PESSOA, 2001; SOUZA; GARCIA, 2019).

Apesar de muitos docentes afirmarem reconhecer a importância e a necessidade da realização de atividades práticas em sala de aula, na maioria das vezes, isso não acontece (MEGID NETO; FRACALANZA, 2003). Os professores dizem encontrar muitos empecilhos na realização de aulas menos tradicionais, desde a falta de estrutura física na escola, falta de material didático, número reduzido de aulas, grande número de alunos por sala, até a necessidade de alguém que os auxilie na organização das aulas laboratoriais (MEGID NETO; FRACALANZA, 2003; NASCIMENTO et al., 2017).

Mesmo que se considere o uso de atividades práticas como uma ferramenta positiva para o ensino, é necessário que se atente também para a formação dos profissionais de educação, em que, muitas vezes, falta a familiarização com os assuntos, a mediação pedagógica e o questionamento dos velhos paradigmas educacionais. Todos esses problemas, se não forem repensados e modificados, contribuem para que não sejam elaboradas atividades inovadoras, fazendo com que se mantenha a forma mecânica como os conteúdos dos livros didáticos são transmitidos (CECCANTINI, 2006; SILVA; GHILARDI-LOPES, 2014).

De acordo com Oliveira (2010) e Souza e Garcia (2019), as aulas experimentais são atrativas por se esperar que possam trazer diversas contribuições no ensino e aprendizagem de ciências, como: motivar e despertar a atenção dos alunos, desenvolver a capacidade de trabalhar em grupo, desenvolver a iniciativa pessoal e a tomada de decisão, estimular a criatividade, aprimorar a capacidade de observação e registro de informações, aprender a analisar dados e propor hipóteses para os fenômenos e aprender conceitos científicos, entre muitas outras. 
Segundo Dias, Schwarz e Vieira (2009), o estudo das plantas ainda é realizado de maneira desestimuladora e de forma diferente da realidade da escola e da comunidade. Resumem-se em aulas teóricas, em que são utilizadas receitas prontas retiradas de livros didáticos, na maioria das vezes, apresentadas apenas em datas comemorativas, de forma pontual, como por exemplo, o dia da árvore, semana do meio ambiente ou em feira de ciências.

De acordo com Silva et al. (2015), a prática educativa com aula de campo, por exemplo, trabalhada com dedicação, é considerada uma ruptura das atividades realizadas em classe, contribuindo para o aprofundamento dos conteúdos abordados em sala de aula, e para a formação do aluno no campo social, intelectual e afetivo, tornando momentos de aprendizagem inesquecíveis.

Assim, as aulas práticas, como método didático, são decisivas para o aprendizado das Ciências, pois contribuem para a formação científica, tendo em vista que aguça a observação, manipulação e construção de modelo. Elas devem permitir ao estudante observar, vivenciar e discutir conjunto de experiências e fenômenos biológicos e físico-químicos relacionados com seu cotidiano.

Neste contexto, o trabalho objetivou introduzir os alunos do sexto ano do Ensino Fundamental de três Escolas Municipais do Município de São Gabriel, Rio Grande do Sul no universo da ciência com a execução de atividades práticas de Botânica, com produção de material didático alternativo e a implementação de práticas laboratoriais e de campo.

\section{MATERIAL E MÉTODOS}

O presente estudo foi desenvolvido com alunos de três turmas do sexto ano de três Escolas municipais de São Gabriel/RS, localizada na região fisiográfica da Campanha Gaúcha. A cidade possui aproximadamente 65.000 habitantes (IBGE, 2015), e sua economia é voltada principalmente para a agropecuária, sendo a produção de arroz, soja e gado de corte as predominantes. As atividades foram desenvolvidas durante o ano de 2016 por acadêmicos dos Cursos de Bacharelado em Biotecnologia e Licenciatura em Ciências Biológicas da Universidade Federal do Pampa (Unipampa) - Campus São Gabriel.

\subsection{Atividades desenvolvidas nas Escolas Municipais participantes}




\subsubsection{Palestras}

Participaram um total de 40 alunos por palestras e professores das mesmas, totalizando 120 alunos por palestra. Para a realização das palestras, foi utilizado dispositivo audiovisual (Datashow), quadro branco, vidraria de laboratório, cartazes, partes vegetativas e reprodutivas de plantas vivas, vídeos, material (lixo seco) reciclável e não-reciclável, dentro outros. Cada palestra teve duração de 50 a 60 minutos conforme a interação com os alunos.

Os temas abordados foram educação ambiental, vidrarias de um Laboratório de Ciência ou Biologia, células vegetal, animal e procarionte (componentes e funções), vasos condutores das plantas, formas de fermentação, pigmentos vegetais, estruturas vegetativas e reprodutivas de plantas e hormônios vegetais. Para maior compreensão dos temas, buscou-se utilizar linguagem compatível com o desenvolvimento cognitivo dos alunos das Escolas, evitando-se a utilização de termos técnicos. Ainda, a faixa etária dos alunos ficava em 12 a 14 anos de idade.

\subsubsection{Produção de material didático}

A biologia celular, a anatomia e a fisiologia vegetal foram utilizadas como base fundamental para o entendimento dos vegetais na manutenção dos serviços ecológicos nos diferentes ecossistemas. Para isso, foram confeccionados modelos didáticos ampliados com todas as estruturas que, quando analisados, permitem o estudo com maior facilidade. Ademais, com as estruturas anatômicas, o aluno pode analisar as estruturas encontradas tipicamente em cada região da planta, relacionando-as com suas funções (fisiologia vegetal) dentro do vegetal como um todo. Cabe ressaltar que todo o material confeccionado foi disponibilizado para os professores das Escolas envolvidas.

\subsubsection{Experimentos demonstrativos}

Após as palestras, os discentes desenvolveram vários experimentos laboratoriais e no campo envolvendo os assuntos abordados, sendo desenvolvidos em sala de aula ou externamente. 
Os experimentos tinham como objetivo trabalhar os conteúdos de Botânica de forma a despertar a curiosidade dos discentes e promover uma maior integração com os acadêmicos da Unipampa. Ainda, após a apresentação dos experimentos, foi distribuído material informativo para os discentes, os quais continham exemplares que poderiam ser pintados.

Descrição dos experimentos:

- Atividade de fermentação: esta atividade foi realizada em sala de aula com três aulas práticas sobre fermentação alcoólica; no decorrer da aula, o processo de fermentação foi demonstrado e visualizado com apontamentos de dúvidas por parte dos alunos e, com demonstração e visualização da atividade enzimática dessa forma de fermentação. Cabe ressaltar que, durante as palestras realizadas, este tema foi exaustivamente estudado com demonstrações práticas e exemplos.

- Pigmentos vegetais: esta atividade envolveu procedimentos em sala de aula e atividades práticas a campo. No campo, ocorreu visualização dos diferentes pigmentos nos vegetais, especialmente nas folhas e, em sala de aula, foram utilizados extratores para confirmar a presença dos mesmos. Ainda, foi abordado o uso das vidrarias de laboratório nas aulas práticas e, em uma das visitas a Unipampa - Campus São Gabriel, os alunos tiveram a oportunidade de vivenciar aulas práticas no Laboratório de Bioquímica, de Botânica e de Zoologia e, também nas Escolas, os alunos tiveram a oportunidade de manusear e utilizar várias vidrarias.

- Partes vegetativas e reprodutivas das plantas: nesta atividade, foi evidenciada a morfologia vegetal, estudos dos diferentes tipos e classificação de raiz, caule, folha, flor, fruto e semente; a atividade foi desenvolvida com auxílio de material vegetal vivo e com ajuda de lupa estereoscópica.

- Hormônios vegetais: esta atividade foi conduzida a campo e se utilizaram mudas de espécies florestais para representar a atuação dos fitormônios, locais específicos e/ou induzidos por estas macromoléculas. Esta atividade foi desenvolvida nas Escolas e na Casa de Vegetação da Unipampa - Campus São Gabriel.

\section{RESULTADOS E DISCUSSÃO}

A utilização das aulas práticas seja em Laboratório ou de campo vincula experimentos de observação/atuação e complementam os estudos teóricos. 
Os modelos didáticos (Figura 1) foram depositados nas Escolas para serem utilizados pelos professores de Ciências e Biologia e também serviram de modelo para confecção de novos modelos didáticos para os Cursos de Bacharelado em Biotecnologia, Licenciatura e Bacharelado em Ciências Biológicas e Bacharelado em Engenharia Florestal, mais especificamente, para os componentes curriculares Fisiologia Vegetal e Anatomia e Morfologia Vegetal na Universidade Federal do Pampa - Campus São Gabriel.



Figura 1 - Modelos didáticos de células vegetais desenvolvidas por alunos das três Escolas do Município de São Gabriel, RS

Fonte: Autores (2016).

O material produzido pelos alunos, que se revelou bastante rico e, curiosamente, convergente com muitos dos conteúdos sobre as temáticas abordadas, atingiu as perspectivas esperadas mostrando que com pouco custo e material reutilizável é possível realizar aulas para que ocorra a assimilação completa dos conteúdos.

A frequência nas palestras e aulas em sala de aula e/ou campo foi considerada satisfatória com participação ativa no desenvolvimento das atividades práticas, manuseando as amostras de vegetais (folhas, caules e raízes), preparando alguns experimentos e conversando a respeito do tema abordado. Foi observado grande entusiasmo e curiosidade por parte dos alunos durante a realização das atividades.

Esse interesse por parte dos alunos nas explanações também foi observado por Falcão e Falcão Sobrinho (2014) que comentaram em seu trabalho que os discentes participaram com indagações, perguntas, demonstrando sensações de espanto e empolgação, bem como de curiosidade frente a novos recursos didáticos utilizados (exposição de pinturas, experimentos, exposição de maquetes, etc.). 
As aulas práticas que foram especificamente de campo (Figura 2), atividades de visualização e aplicabilidade de hormônios vegetais, permitiram o desenvolvimento no aluno da atenção em relação à diversidade da natureza, especialmente na atuação dos diferentes hormônios vegetais, facilitando a observação e comparação, as quais, segundo Ferrara (2001) e Albuquerque e Zárate (2017), as aulas práticas de campo orientam o desenvolvimento da atenção.

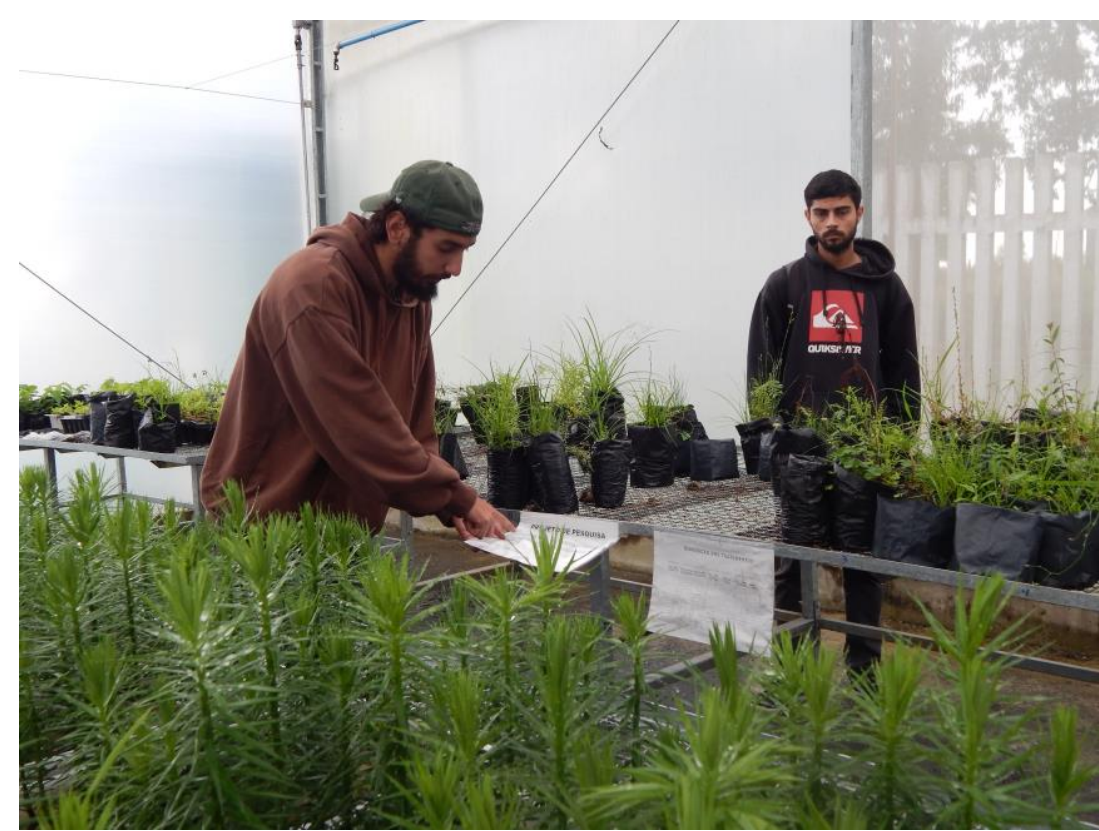

Figura 2 - Aula prática de campo na Casa de Vegetação da Universidade Federal do Pampa - Campus São Gabriel Fonte: Autores (2016).

Adicionalmente Cornacini et al. (2017) enfatizam que a importância das atividades de campo, enquanto instrumentos no processo de ensino e aprendizagem, pode ser encontrada dispersa em publicações relacionadas aos diferentes períodos de nossa história educacional. Com efeito, aulas de campo voltadas para conceitos e conteúdos específicos de algumas disciplinas como a Biologia, mas especificamente, com a Botânica, associadas às atividades de estudo do meio, tendem a gerar interesse crescente para essa modalidade de atividade educativa.

Além da utilização de palestras e/ou oficinas, a atividade experimental também favorece o processo de ensino-aprendizagem, pois aproxima o discente de seu cotidiano. Ao serem apresentados aos experimentos, os discentes se tornam mais ativos e mais interessados no tema a ser estudado, fazendo reflexões e facilitando o aprendizado (WEBER et al., 2017). Ainda, antes da montagem e realização de alguns experimentos (pigmentos vegetais, 
fermentação e enzimas) questionavam-se os discentes sobre o que eles achavam ou quais eram suas expectativas a respeito do que poderia vir a ocorrer em cada situação. Foi observado que estes questionamentos instigavam as crianças e estas ficavam ainda mais atentas.

Vários estudos têm afirmado à importância da utilização de formas de ensino de Botânica que utilizem o diálogo e a experiência prática, bem como pode ser a base para a compreensão do ambiente. Biondi e Falkowski (2009) observaram o aumento do conhecimento dos discentes de 8 a 10 anos, da cidade de Curitiba/PR, sobre solos após atividades de educação ambiental com explanações e cartazes. Já Gordin et al. (2013) citam que atividades como pintura com solo, manuseio de materiais e pequenas excursões pelo município de Dourados/MS motivaram discentes com relação ao tema solo.

Silva e Ferrari (2012) observaram que atividades mais participativas e interativas promoveram reflexão, sensibilização e conscientização a respeito da importância da conservação de recursos naturais como o solo, água e vegetação. Além disso, esses pesquisadores descrevem que as oficinas e aulas práticas têm grande receptividade pelos discentes, sendo que estes apresentam alegria, entusiasmo e motivação durante o desenvolvimento das atividades. Também, Pereira e Putzke (1996) enfatizam que uma aula de campo não se refere apenas em visitas a matas ou florestas, mas a qualquer ambiente diferente de sala de aula, podendo inclusive ser o pátio da escola, ruas do bairro ou parques, lugares onde os estudantes podem ser motivados a participarem das ações.

Observou-se que, após o término das aulas de campo, muitos alunos surgiram com curiosidades do conteúdo dado, demonstrando assim maior interesse pela temática, confirmando que, com a utilização dessas práticas, os alunos assimilaram melhor os conhecimentos adquiridos, uma vez que melhorou a compreensão da temática, muitas vezes considerada "cansativa", se mostrando mais branda e maleável em prática.

Ainda, cabe ressaltar que foi obtido um retorno por parte das Escolas envolvidas enfatizando que, comparando com anos anteriores, constatou-se que os estudantes obtiveram um conhecimento científico mais avançado por meio dessa nova experiência, facilitando o contato com a natureza e com os materiais biológicos que utilizaram desenvolvendo assim algumas habilidades científicas práticas como observar e manipular as plantas e os materiais de Laboratório de Química, de Bioquímica e de Biologia, bem como na interpretação dos resultados obtidos. Desta forma, o trabalho prático concebido como uma atividade de 
investigação de campo promoveu um alargamento do conhecimento científico por parte dos alunos.

A utilização de diferentes procedimentos de ensino pode fomentar uma atitude reflexiva por parte do aluno, na medida em que ofereça a este oportunidades de participação, nas quais, vivencie uma variedade de experiências, seja solicitado a tomar decisões, fazer julgamentos e chegar a conclusões. Cabe ao professor a responsabilidade de articular as diferentes modalidades didáticas para que tais objetivos possam ser alcançados (ROTTA; CARVALHO; BETRAMI, 2008). Ainda, pode-se observar, neste estudo, que as atividades realizadas são positivas para o processo de ensino-aprendizagem, visto que o conhecimento construído tem significado para o discente (WEBER et al., 2017). As atividades desta natureza deveriam se tornar cotidianas em todas as escolas de Ensino Fundamental e Médio.

A experimentação no ensino de Ciências e de Biologia favorece a construção de relações entre a teoria e a prática, bem como uma relação entre as concepções prévias que cada aluno já traz consigo e as novas ideias que estão sendo trabalhadas. Nas aulas práticas, os alunos têm a oportunidade de desenvolver habilidades e competências, atitudes e valores, bem como construir e reconstruir conceitos de forma ativa (CAVALCANTE; SILVA, 2008). As atividades práticas, especificamente no conteúdo de Botânica, visam incentivar uma metodologia que valorize a compreensão e a interpretação da natureza (KRASILCHIK, 1996).

Desta forma, o aluno passa a ter condições para compreender, de uma forma mais ampla, determinados conceitos, além de desenvolver habilidades e competências que facilitam seu entendimento do mundo em que vive; daí a necessidade de se estabelecer uma relação entre o cotidiano do aluno e o que é trabalhado em sala de aula (NANNI, 2004). Segundo Silva e Moraes (2011) e Souza e Garcia (2019), o professor desempenha um papel fundamental no ensino das disciplinas curriculares de suas turmas, mas também na formação de cidadãos; é ele que participa de forma ativa no processo de crescimento intelectual de seus alunos, reforçando a necessidade de busca por alternativas metodológicas que possam contribuir com a aprendizagem, conduzindo aquele que aprende à compreensão da realidade em que está inserido.

Durante esse trabalho, buscou-se tornar o ensino da Botânica mais consistente e prazeroso para discentes. O material elaborado visou abranger diversos conteúdos e tornar as aulas mais dinâmicas. Além disso, os modelos didáticos são materiais permanentes, o que faz com que estejam sempre disponíveis para novos alunos e professores, despertando interesse 
de confeccionar novas estruturas que venha auxiliar nas aulas teóricas e práticas nas Escolas. Cabe ressaltar a importância de realizar trabalhos entre a Universidade com as Escolas, por ser uma forma de externar, compartilhar e adquirir conhecimento e, aproximar a comunidade local à Universidade.

\title{
4 CONSIDERAÇÕES FINAIS
}

As crianças com as quais foi desenvolvido este trabalho já possuíam alguns conhecimentos relacionados à Botânica, principalmente no que se refere a sua importância para a vida do homem, os quais foram demonstrados, com as atividades propostas no trabalho, com ótimo desenvolvimento nas aulas práticas e na construção dos modelos didáticos.

Os materiais didáticos produzidos durante o trabalho foram disponibilizados às Escolas para serem utilizados para facilitar o aprendizado do aluno e auxiliar os professores em aulas práticas diversificadas.

Os acadêmicos da Universidade Federal do Pampa, monitores do projeto, também encontraram grande satisfação na realização das atividades junto aos discentes, pois para a maioria, esta foi a primeira experiência com crianças. Para os acadêmicos do curso de Bacharel em Biotecnologia e Licenciatura em Ciências Biológicas, esta foi uma experiência importante para sua formação profissional, visto que estes profissionais podem trabalhar com educação ambiental, especialmente os do Curso de Licenciatura em Ciências Biológicas.

\section{PRACTICAL CLASSES AS A STRATEGY FOR BOTANICAL TEACHING IN MIDDLE SCHOOL}

\begin{abstract}
In the teaching of science, Botany is one of the areas with the greatest difficulty in assimilating content. Among the causes, we highlight the students' lack of interest in the theme, the lack of practical classes and suitable materials. This study aimed to evaluate the performance of lectures and experiments on the learning of Botany concepts by elementary school students. The present study took place, in the city of São Gabriel in the state of Rio Grande do Sul, in three public schools. The classes were taught and performed at the laboratory and also in its own field, with the participation of 120 students in each activity. It could be observed in this study that the activities performed were positive for the teachinglearning process, with increased knowledge; since, the knowledge was significant for the students of the Elementary School as for the students of the Universidade Federal of Pampa,
\end{abstract}


especially for their professional formation, especially those of the Degree in Biological Sciences.

Keyword: Didactic materials. Environmental education.Teaching-learning.

\section{REFERÊNCIAS}

ALBUQUERQUE, Janaína Vital de; ZÁRATE, Eliete Lima de Paula. Materiais didáticos de botânica criptogâmica muito além dos livros: entrelaçando os saberes na graduação.

Experiências em Ensino de Ciências, Porto Alegre, v. 12, n. 8, p. 239-249, 2017. Disponível em: http://if.ufmt.br/eenci/artigos/Artigo_ID448/v12_n8_a2017.pdf. Acesso em: 23 mar. 2019.

BIONDI, Daniela; FALKOWSKI, Vanessa. Avaliação de uma atividade de educação ambiental com o tema "solo". Revista Eletrônica do Mestrado em Educação Ambiental, Rio Grande, v. 22, p. 202-215, 2009. Disponível em: https://periodicos.furg.br/. Acesso em: 19 fev. 2019.

CAVALCANTE, Dannuza Dias; SILVA, Aparecida de Fátima Andrade da. Modelos didáticos de professores: concepções de ensino-aprendizagem e experimentação. In: ENCONTRO NACIONAL DE ENSINO DE QUÍMICA, XIV., 2008, Curitiba. Anais [...]. Curitiba: UFPR, 2008.

CECCANTINI, Gregório. Os tecidos vegetais têm três dimensões. Revista Brasileira de Botânica. São Paulo, v. 29, n. 2, p. 335-337, 2006. Disponível em: http://www.scielo.br/pdf/rbb/v29n2/a15v29n2.pdf. Acesso em: 23 mar. 2019.

CORNACINI, Maiara Ribeiro; SILVA, Renan Gonçalves da; DORNFELD, Carolina Buso; CAMARGOS, Liliane Santos de. Percepção de alunos do ensino fundamental sobre a temática botânica por meio de atividade experimental. Experiências em Ensino de Ciências, Porto Alegre, v. 12, n.4, p. 166-184, 2017. Disponível em: https://if.ufmt.br/eenci/artigos/Artigo_ID375/v12_n4_a2017.pdf. Acesso em: 23 mar. 2019.

DIAS, Jane Maria de Castro; SCHWARZ, Elizabeth de Araújo; VIEIRA, Eliane do Rocio. A botânica além da sala de aula. 2009. 1-21 p.

FALCÃO, Cleire Lima da Costa; FALCÃO SOBRINHO, José. A utilização de recursos didáticos como auxiliares no processo de aprendizagem do solo. Revista da Casa da Geografia de Sobral, Sobral, v. 16, n. 1, p-. 19-28, 2014. Disponível em: http://rcgs.uvanet.br/index.php/RCGS/article/view/156. Acesso em: 23 abr. 2019.

FERRARA, Lucrécia D’Aléssio. Leitura sem palavras. São Paulo: Ática. 2001. 72 p.

GORDIN, Carla; PAGLIARINI, Maximiliano Kawahata; SANTOS, Adriana Marques; BRANDÃO NETO, João Freitas; PEIXOTO, Paula Pinheiro Padovese. Inserindo o solo na educação ambiental: uma experiência no Centro-Oeste. Revista Agrarian, Dourados, v. 6, p. 
97-106, 2013. Disponível em: https://ojs.ufgd.edu.br/index.php/agrarian/article/view/1653/. Acesso em: 19 mar. 2019.

IBGE - Instituto Brasileiro de Geografia e Estatística. IBGE Cidades: São Gabriel. Rio de Janeiro: IBGE, 2015. Disponível em:

http://www.cidades.ibge.gov.br/xtras/perfil.php?lang=\&codmun=431830\&search=riogranded o-sul|saogabriel|infograficos:-informacoes-completas. Acesso em: 15 jan. 2019.

KRASILCHICK, Myriam. Prática de biologia. São Paulo: Edusp. 1996.

NANNI, Roberto. A natureza do conhecimento científico e a experimentação no ensino de ciências. Revista Eletrônica de Ciências, São Carlos, v. 26, p. 53-67, 2004.

NASCIMENTO, Beatriz Miguez; DONATO, Ana Maria; SIQUEIRA, Andréa Espinola de; BARROSO, Carolina Burlamaqui; SOUZA, Antônio Carlos Teixeira; LACERDA, Silvana Messere de; BORIM, Danielle Cristina Duque Estrada. Propostas pedagógicas para o ensino de botânica nas aulas de ciências: diminuindo entraves. Revista Electrónica de Enseñanza de las Ciencias, v. 16, n. 2, p. 298-315, 2017. Disponível em:

http://reec.uvigo.es/volumenes/volumen16/REEC_16_2_7_ex1120.pdf. Acesso em: 08 abr. 2019.

MEGID NETO, Jorge; FRACALANZA, Hilário. O livro didático de ciências: problemas e soluções. Ciência \& Educação, Bauru, v. 9, n. 2, p. 147-157, 2003. Disponível em: http://www.scielo.br/pdf/ciedu/v9n2/01.pdf. Acesso em: 23 mar. 2019.

OLIVEIRA, Jane Raquel Silva de. Contribuições e abordagens das atividades experimentais no ensino de ciências: reunindo elementos para a prática docente. Acta Scientiae, Canoas, v. 12, n. 1, p. 139-153, 2010. Disponível em: http://w3.ufsm.br/laequi/wpcontent/uploads/2015/03/contribui\%C3\%A7\%C3\%B5es-e-abordagens-de-atividadesexperimentais.pdf. Acesso em: 19 fev. 2019.

PEREIRA, Antônio Batista; PUTZKE, Jair. Ensino de botânica e ecologia: proposta metodológica. Porto Alegre: Sagra-Luzzatto, 1996. 184p.

PESSOA, Oswaldo Frota. Os caminhos da vida. São Paulo: Scipione. 2001. 548 p.

ROTTA, Emilio; CARVALHO, Lucas Caminha de; BELTRAMI, Marlise Zonta. Manual de prática de coleta e herborização de material botânico. Colombo: Embrapa Florestas. 2008.

SILVA, Aline Gomes Fernandes da; FERRARI, Jéferson Luiz. A oficina pedagógica no ensino fundamental como estratégia de ensino-aprendizagem para conservação do solo e da água. Revista Verde de Agroecologia e Desenvolvimento Sustentável, Pombal, v. 7, n. 5, p. 107-112, 2012. Disponível em:

https://www.gvaa.com.br/revista/index.php/RVADS/article/view/1476. Acesso em: 23 mar. 2019.

SILVA, Ana Paula Miranda da; SILVA, Maria Francilene Souza; ROCHA, Francinalda Maria Rodrigues da; ANDRADE, Ivanilza Moreira de. Aulas práticas como estratégia para o 
conhecimento em botânica no ensino fundamental. Holos, Natal, v. 31, n. 8, p. 68-79, 2015. Disponível em: http://www2.ifrn.edu.br/ojs/index.php/HOLOS/article/view/2347. Acesso em: 23 mar. 2019.

SILVA, Audilia Borges Vitorina; MORAES, Moemy Gomes. Jogos pedagógicos como estratégia no ensino de morfologia vegetal. Revista Enciclopédia Biosfera - Centro Científico Conhecer, Goiânia, v. 7, n. 13, p. 1642-1651, 2011.

SILVA, Fabrícia Souza da; TERÁN, Augusto Fáchin. Práticas pedagógicas na educação ambiental com estudantes do ensino fundamental. Experiências em Ensino de Ciências, Porto Alegre, v. 13, n. 5, p. 340-351, 2018. Disponível em:

http://if.ufmt.br/eenci/artigos/Artigo_ID543/v13_n5_a2018.pdf. Acesso em 08 abr. 2019.

SILVA, Juliana Nascimento; GHILARDI-LOPES, Natalia Pirani. Botânica no ensino fundamental: diagnósticos de dificuldades no ensino e da percepção e representação da biodiversidade vegetal por estudantes. Revista Electrónica de Enseñanza de las Ciencias, v. 13, n. 2, p. 115-136, 2014. Disponível em:

http://reec.uvigo.es/volumenes/volumen13/REEC_13_2_1_ex773.pdf. Acesso em: 23 mar. 2019.

SOUZA, Cássia Luã Pires de; GARCIA, Rosane Nunes. Uma análise do conteúdo de botânica sob o enfoque Ciência-Tecnologia-Sociedade (CTS) em livros didáticos de biologia do ensino médio. Ciência \& Educação, Bauru, v. 25, n. 1, p. 111-130, 2019. Disponível em:

http://www.scielo.br/pdf/ciedu/v25n1/1516-7313-ciedu-25-01-0111.pdf. Acesso em: 23 mar. 2019.

SOUZA, Cássia Luã Pires de; KINDEL, Eunice Aita Isaia. Compartilhando ações e práticas significativas para o ensino de botânica na educação básica. Experiência em Ensino de Ciências, Porto Alegre, v. 9, n. 3, p. 44-58, 2014. Disponível em: http://if.ufmt.br/eenci/artigos/Artigo_ID253/v9_n3_a2014.pdf. Acesso em: 19 fev. 2019.

WEBER, Mirla Andrade; FRANÇA, Jéssica Silveira; FELIPPE, Bruno Moreira; FLORES, Josiane Martins; LORENTZ, Leandro Homrich; VIEIRA, Frederico Costa Beber.

Ferramentas úteis para o aprendizado em solos de estudantes do quarto ano do ensino fundamental. Experiência em Ensino de Ciências, Porto Alegre, v. 12, n. 3, p. 69-79, 2017. Disponível em: http://if.ufmt.br/eenci/?go=artigos\&idEdicao=55. Acesso em: 19 abr. 2019.

\section{DADOS DOS AUTORES}

\section{Bruna Borges Trindade}

E-mail: bru.tborges@gmail.com

Currículo Lattes: http://lattes.cnpq.br/7121006033813472

Doutoranda em Ciências Biológicas, mestrado em Ciências Biológicas e graduação em Biotecnologia (Bacharelado) pela Universidade Federal do Pampa (UNIPAMPA) - Campus São Gabriel. Tem experiência na área de Neurobiologia com ênfase em Toxinologia, Eletrofisiologia e Bioquímica e, é integrante do Laboratório de Neurobiologia e Toxinologia 
(LANETOX) da UNIPAMPA - Campus São Gabriel atuando na pesquisa de toxinas animais e vegetais com propriedades inseticidas.

\section{Júlia Dutra Vargas}

E-mail: julinhadv2@ hotmail.com

Currículo Lattes: http://lattes.cnpq.br/4941301411695400

Possui Graduação em Biotecnologia (Bacharelado) pela UNIPAMPA - Campus São Gabriel. Tem experiência na área de Farmácia, com ênfase em análise toxicológica.

\section{Paula Juliane Barbosa de Oliveira}

Currículo Lattes: http://lattes.cnpq.br/9184213171069125

E-mail: jusg.paula.unibiotec@gmail.com

Doutoranda em Agronomia pela Universidade Federal Tecnológica do Paraná (UTFPR) Campus Pato Branco. Mestrado em Biotecnologia pela Universidade Tecnológica Federal do Paraná (UTFPR) - Campus Dois Vizinhos. Graduação em Biotecnologia (Bacharelado) pela UNIPAMPA - Campus São Gabriel. Tem experiência em microbiologia ambiental, microbiologia agrícola, biologia molecular e fitotecnia.

\section{Silvane Vestena}

E-mail: silvane.vestena@ifmg.edu.br

Currículo Lattes: http://lattes.cnpq.br/7239967672848394

Doutorado em Fisiologia Vegetal pela Universidade Federal de Viçosa (UFV), mestrado em Botânica pela Universidade Federal do Rio Grande do Sul (UFRGS) e graduação em Ciências Biológicas pela Universidade Federal de Santa Maria (UFSM). Atualmente é docente na Universidade Federal do Pampa (UNIPAMPA) - Campus São Gabriel. Tem experiência em Nutrição Mineral em Plantas e Metabolismo Vegetal com ênfase em fitorremediação de metais pesados, propagação de plantas e toxinas vegetais com propriedades inseticidas. 